\title{
Chelation-Assisted C-O B ond Cleavage of Ortho Esters. A Convenient Synthesis of myo-I nositol Derivatives Having Free Hydroxy Group(s) at Specific Position(s)
}

\author{
Sue-Min Yeh, Gene Hsiang Lee, Yu Wang, and Tien-Yau Luh* \\ Department of Chemistry, National Taiwan University, Taipei, Taiwan 106, Republic of China
}

Received April 2, $1997^{\otimes}$

\begin{abstract}
Reactions of ortho esters of myo-inositol $\mathbf{8}$ or $\mathbf{1 0}$ with 1-2 equiv of Grignard reagents in benzeneether yield regio- and stereoselectively the corresponding ring opening products having a free hydroxy group at C-1. The regiosel ectivity is rationalized owing to the presence of the 2-methoxy group which will serve as an auxiliary to form a chelation complex $\mathbf{1 2}$ with magnesium. Inositol derivatives having two free hydroxy group at C-1 and C-3 positions can be achieved from reactions of $\mathbf{6}$ or $\mathbf{8}$ with excess Grignard reagents or under more drastic conditions. The reaction of $\mathbf{8 b}$ with excess $\mathrm{LiAlH}{ }_{4} / \mathrm{AlCl}_{3}$, on the other hand, yields the corresponding 1,5-diol 19.
\end{abstract}

Inositol derivatives are important biologically. ${ }^{1}$ Tremendous efforts have been endeavored in synthesizing partially protected inositols having the free hydroxy group(s) at specific position(s). Acetal functionalities are most commonly used to protect vicinal hydroxy groups in inositols.1,2 To illustrate this, acetals $\mathbf{1}$ to $\mathbf{4}$ have widely been employed as the starting materials for the synthesis of various derivatives of myo-inositols. ${ }^{3,4}$ However, multistep synthesis and tedious separation of regioisomers are frequently required to access $2-\mathbf{4}$. More recently, Kishi and several other groups have employed orthoformate of myo-inositol $\mathbf{5}$ for the preparation of certain inositol derivatives having one to three free hydroxy groups. ${ }^{3}$

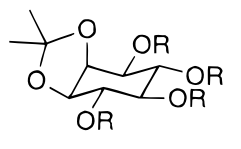

1

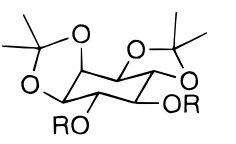

2

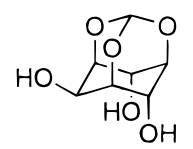

4

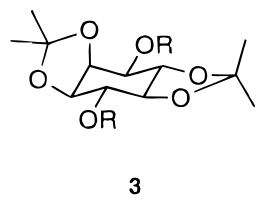

5
It is well documented that nucleophiles can react with acetals to give the corresponding alkoxy alcohols. ${ }^{4}$ Reaction of $\mathbf{6}$ with $\mathrm{Me}_{3} \mathrm{Al}$ has been shown to afford $\mathbf{7}$ selectively (eq 1). 3 e,f We recently uncovered a chelationassisted regioselective ring opening of acetals with Grig-

${ }^{\otimes}$ Abstract published in Advance ACS Abstracts, November 1, 1997. (1) For reviews, see: (a) David, S.; Hanessian, S. Tetrahedron 1985, 41, 643. (b) Billington, D. C. Chem. Soc. Rev. 1989, 18, 83. (c) Potter, B. V. L. Nat. Prod. Rep. 1990, 1. (d) Beaucage, S. L.; I yer, R. P. Tetrahedron 1993, 49, 10441. (e) Billington, D. C. The Inositol PhosphateChemical-Synthesis and Biological Significance VCH: New York, 1993. (f) Potter, B. V. L.; Lampe, D. Angew. Chem., Int. Ed. Engl. 1995, 34, 1933. (g) Kiddle, J J . Chem. Rev. (Washington, D.C.) 1995, 95, 2189. (h) Hudlicky, T.; Cebulak, M. Cyclitols And Their Derivatives: A Handbook of Physical, Spectral, and Synthetic Data; $\mathrm{VCH}$ : New York, 1993.

(2) (a) Greene, T. A.; Wuts, P. G. M. Protective Groups in Organic Synthesis, 2nd ed.; Wiley: New York, 1991. (b) Kocienski, P. J . Protective Groups; Thieme: New York, 1994. nard reagents (eq 2). ${ }^{4 d, e}$ We felt that this reaction can be extended to the ortho esters of inositols to selectively give inositol derivatives having one or two free hydroxy groups at certain specific position(s).

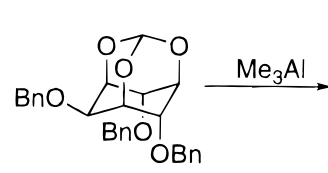

6

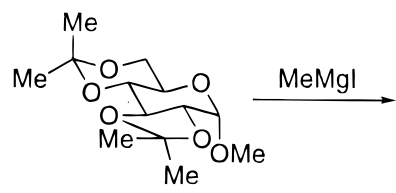<smiles>C[C@H]1OC2C(Br)OC(C(OCc3ccccc3)C2OCc2ccccc2)C1O</smiles>

7

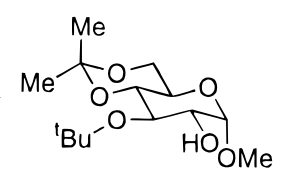

Inositol derivative 8a was treated with $\mathrm{MeMgl}$ (2 equiv) in refluxing benzene-ether (10:3) for $16 \mathrm{~h}$ to give the corresponding ring opening derivative $9 \mathrm{a}$ in $83 \%$ yield. ${ }^{5}$ Similar reactions with $\mathrm{EtMgBr}$ and $\mathrm{PhMgBr}$ gave $\mathbf{9 b}$ and $\mathbf{9 c}$ in $68 \%$ yield each (eq 3 ). ${ }^{5}$ When more reactive $\mathbf{8 b}$ was employed, the reaction was carried out at room temperature in the same medium to afford $\mathbf{9 d}$ in $57 \%$ yield. ${ }^{5}$ The stereochemistry was determined by 2D NOESY experiments and by the X-ray structure of 9d. ${ }^{8}$ The nucleophile apparently attacks from the opposite site to the $\mathrm{C}-\mathrm{O}$ bond being cleaved. Presumably, due to the rigidity of the skeleton of $\mathbf{6}$ or $\mathbf{8}$, cleavage of the $\mathrm{C}-\mathrm{O}$ bond with inversion of configuration was observed.

(3) (a) Vogl, O.; Anderson, B. C.; Simons, D. M. J . Org. Chem. 1969 34, 204. (b) Lee, H. W.; Kishi, Y.; J . Org. Chem. 1985, 50, 4402. (c) Billington, D. C.; Baker, R. J . Chem. Soc., Chem. Commun. 1987, 1011. (d) Baudin, G.; Glänzer, B. I.; Swaminathan, K. S.; Vasella, A. Helv. Chim. Acta 1988, 71, 1367. (e) Gilbert, I. H.; Holmes, A. B.; Young, R. C. Tetrahedron Lett. 1990, 31, 2633. (f) Gilbert, I. H.; Holmes, A. B.; Pestchanker, N. J .; Young, R. C. Carbohyd. Res. 1992, 234, 117. (g) Andersch, P.; Schneider, M. P. Tetrahedron: Asymmetry 1993, 4, 2135 (h) Li, C.; Vasella, A. Helv. Chim. Acta 1993, 76, 211. (i) Banerjee, T.; Srikantiah, S. M. Tetrahedron Lett. 1994, 35, 8053. (j) Ozaki, S.; Koya, Y.; Ling, L.; Watanabe, Y.; Kimura, Y.; Hirata, M. Bull. Chem. Soc., J pn. 1994, 67, 1058.

(4) For reviews, see: (a) Trofimov, B. A.; Korostova, S. E. Russ. Chem. Rev. 1975, 44, 41. (b) Mukaiyama T.; Murakami, M. Synthesis 1987, 1043. (c) Alexakis A.; Mangeney, P. Tetrahedron: Asymmetry 1990, 1, 477. (d) Luh, T.-Y. Pure Appl. Chem. 1996, 68, 635. (e) Luh, T.-Y. Synlett 1996, 201.

(5) These products were obtained as racemic mixture. 


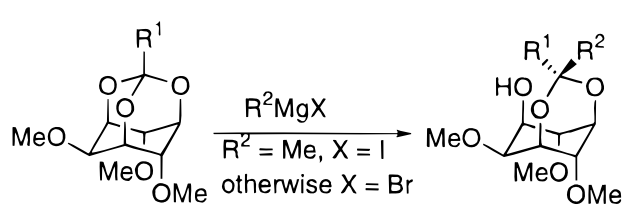

$8 \mathbf{a} R^{1}=H$ $8 b R^{1}=P h$

9a $R^{1}=H, R^{2}=M e \quad 83 \%$ $9 b R^{1}=H, R^{2}=E t \quad 68 \%$ $9 c R^{1}=H, R^{2}=P h \quad 68 \%$ 9d $R^{1}=P h, R^{2}=$ Me $57 \%$

Treatment of $\mathbf{1 0}$ with $\mathrm{MeMgl}$ under the same conditions gave racemic 11a as the sole product (eq 4). The benzylic acetal moiety in $\mathbf{1 0}$ remained intact under these conditions. The structure of 11a was proved by theX-ray crystallography of the corresponding ether $\mathbf{1 1 b} .^{5}$

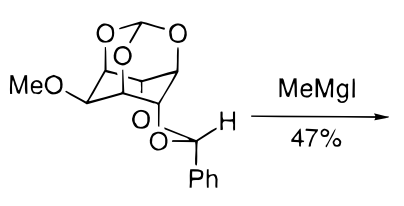

10

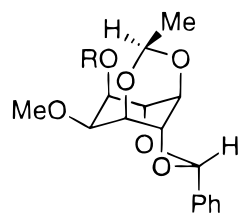

$11 a \mathrm{R}=\mathrm{H}$ $11 \mathrm{~b} R=\mathrm{Me}$
It is noteworthy that the reaction is both regio- and diastereoselective. The regioselectivity of this reaction is understandable owing to the presence of the 2-methoxy group which will serve as an auxiliary to form a chelation complex 12 with magnesium such that selective ring opening is expected. We have tested this viewpoint by investigating the related reaction of scyllo-inositol derivative $\mathbf{1 3}$ with $\mathrm{MeMgl}$ at room temperature. A diastereomeric mixture of $\mathbf{1 4}$ (2:1) was obtained in 56\% yield. No $\mathrm{C}-\mathrm{O}$ bond cleavage at the ortho ester moiety in $\mathbf{1 3}$ was observed. The methoxy group in $\mathbf{1 3}$ apparently directs

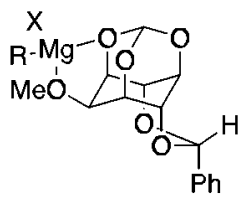

12

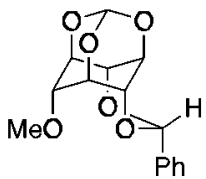

13

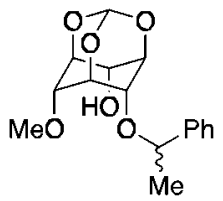

14 the selectivity of the ring opening reaction at the benzylidene moiety. Interestingly, the reaction is nonstereoselective and is compatible with our earlier work on the alkylative ring opening of acetals with the methyl Grignard reagent. ${ }^{6}$

Acetal functionality are known to undergo chelationassisted regioselective ring opening reactions with Grignard reagents. ${ }^{4 d, e, 6}$ The products shown in eqs 3 and 4 contain such acetal group. We felt that a one-pot process may be developed such that a convenient synthesis of inositol derivatives having two free hydroxy group at C-1 and $\mathrm{C}-3$ positions can be achieved from 6 or $\mathbf{8}$. Accordingly, treatments of $\mathbf{6}$ with 3 equiv of MeMgl and with an excess amount of $\mathrm{PhMgBr}$ at refluxing benzene temperature yiel ded the corresponding diols $\mathbf{1 5} \mathbf{a}$ and $\mathbf{1 5 b}$ in $69 \%$ and $72 \%$ yields, respectively. Compound $\mathbf{8 a}$ behaved similarly to give $15 \mathrm{c}$ in $60 \%$ yield (eq 5 ). ${ }^{13} \mathrm{C}$ NMR data (8 signals for $\mathbf{1 5 a}$ and 7 signals for $\mathbf{1 5 b , c}$ due to the absorptions of $\mathrm{sp}^{3}$ carbons) for $\mathbf{1 5}$ are consistent with a $\mathrm{C}_{\mathrm{s}}$ symmetry.

(6) Yuan, T.-M.; Yeh, S.-M.; Hsieh, Y.-T.; Luh, T.-Y. J . Org. Chem. 1994, 59, 8192.<smiles>[R9]C1C2OC3OC(O2)C(O)C1O3</smiles>

$6 \mathrm{R}^{1}=\mathrm{Bn}$

$8 a R^{1}=M e$

$$
\begin{aligned}
& \frac{\mathrm{R}^{2} \mathrm{MgX}}{\mathrm{R}^{2}=\mathrm{Me}, \mathrm{X}=\mathrm{I}} \\
& \text { or } \mathrm{R}^{2}=\mathrm{Ph}, \mathrm{X}=\mathrm{Br}
\end{aligned}
$$$$
\overbrace{\mathrm{OH}} \mathrm{OR}^{1} \mathrm{OR}^{-} \mathrm{CHR}^{2}
$$$$
15 \text { a } R^{1}=B n, R^{2}=M e
$$$$
\text { b } \mathrm{R}^{1}=\mathrm{Bn}, \mathrm{R}^{2}=\mathrm{Ph}
$$$$
c R^{1}=M e, R^{2}=P h
$$

Compounds $\mathbf{1 5}$ appeared to be important intermediates to furnish various inositol derivatives having one to three free hydroxy group at different positions. For example, diacetate $\mathbf{1 6}$ was transformed upon hydrogenolysis into $\mathbf{1 7}$ in $86 \%$ yield.

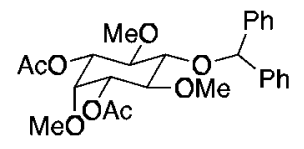

16

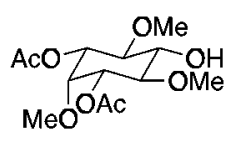

17

Although the 1,5-dihydroxy derivative can be obtained from 9 by protection of the hydroxy group followed by hydrolysis of the acetal moiety (e.g. 9a to 18, eq 6), a more direct synthesis would be desirable. The strategy was based on our previous work using $\mathrm{LiAlH}{ }_{4} / \mathrm{AlCl}_{3}$ to effect regioselective ring opening of acetals. ${ }^{7}$ Thus, reaction of $\mathbf{8 b}$ with an excess of $\mathrm{LiAlH}{ }_{4} / \mathrm{AlCl}_{3}$ for $16 \mathrm{~h}$ afforded $19 \mathrm{in}$ $49 \%$ yield (eq 7). The ${ }^{13} \mathrm{C}$ NMR spectrum of 19 exhibited 10 signals due to absorptions of $\mathrm{sp}^{3}$ carbons which is consistent with the structure with free hydroxy groups at $\mathrm{C}_{1}$ and $\mathrm{C}_{5}$. The discrepancy in regiosel ectivity between the Grignard reagent and the aluminum hydride reagent in these ring opening reactions, however, remains unclear.

$$
\begin{aligned}
& \text { 9a } \underset{\substack{\text { 2. } \mathrm{PTSA}, \mathrm{MeOH} \\
76 \%}}{\mathrm{MeO}, \mathrm{MMe}} \\
& 18
\end{aligned}
$$

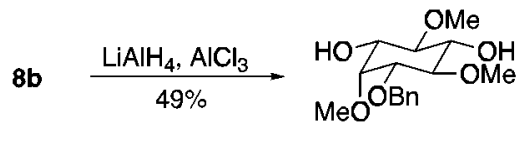

19
(6)

In summary, we have demonstrated a useful method for the selective ring opening of ortho esters of myoinositol derivatives. The procedure described here can serve as a convenient route for the synthesis of myoinositol derivatives having one hydroxy group at C-1 or $\mathrm{C}-5$, and two hydroxy groups at C-1,3 and at C-1,5. Other derivatives could easily be accessed from these intermediates.

\section{Experimental Section}

exo-3,5-O-E thylidene-2,4,6-tri-O-methyl-myo-inositol (9a). Compound $\mathbf{8 a}(3.58 \mathrm{~g}, 15.4 \mathrm{mmol})$ in benzene $(50 \mathrm{~mL})$ was treated with $\mathrm{MeMgl}(15 \mathrm{~mL}$ in $2.05 \mathrm{M}$ ether solution, 30.8 $\mathrm{mmol}$ ). The mixture was heated at ca. $60^{\circ} \mathrm{C}$ for $16 \mathrm{~h}$, cooled,

(7) Yuan, T.-M.; Hsieh, Y.-T.; Yeh, S.-M.; Shyue, J .-J .; Luh, T.-Y. Synlett 1996, 53.

(8) The author has deposited atomic coordinates for this structure with the Cambridge Crystallographic Data Centre. The coordinates can be obtained on request, from the Director, Cambridge Crystallographic Data Centre, 12 Union Road, Cambridge, CB2 1EZ, UK. 
diluted with ether, and washed with saturated $\mathrm{NH}_{4} \mathrm{Cl}$. The organic layer was washed with brine and dried $\left(\mathrm{MgSO}_{4}\right)$. The solvent was removed in vacuo to give the residue which was chromatographed (silica gel, 30\% EtOAc/hexane) to afford racemic $9 \mathrm{a}$ as an oil $\left(3.15 \mathrm{~g}, 83 \%\right.$ ): IR (neat) $v 3503 \mathrm{~cm}^{-1} ;{ }^{1} \mathrm{H}$ NMR $\left(400 \mathrm{MHz}, \mathrm{CDCl}_{3}\right) \delta 1.20(\mathrm{~d}, \mathrm{~J}=4.8 \mathrm{~Hz}, 3 \mathrm{H}), 3.14(\mathrm{~d}, \mathrm{~J}$ $=5.6 \mathrm{~Hz}, 1 \mathrm{H}), 3.33(\mathrm{~s}, 3 \mathrm{H}), 3.47(\mathrm{~s}, 3 \mathrm{H}), 3.49(\mathrm{~s}, 3 \mathrm{H}), 3.48-$ $4.50(\mathrm{~m}, 1 \mathrm{H}), 3.73(\mathrm{dt}, \mathrm{J}=1.2,4.4 \mathrm{~Hz}, 1 \mathrm{H}), 3.79(\mathrm{dd}, \mathrm{J}=6.4$, $7.8 \mathrm{~Hz}, 1 \mathrm{H}$ ), 4.15-4.20 (m, $2 \mathrm{H}), 4.41$ (ddd, J = 2.0, 4.4, 6.4 $\mathrm{Hz}, 1 \mathrm{H}), 5.22(\mathrm{q}, \mathrm{J}=4.8 \mathrm{~Hz}, 1 \mathrm{H}) ;{ }^{33} \mathrm{C} \mathrm{NMR}\left(100 \mathrm{MHz}, \mathrm{CDCl}_{3}\right)$ $\delta$ 21.0, 56.8, 58.0, 58.6, 67.7, 67.9, 71.2, 73.8, 74.9, 84.5, 90.7; HRMS Calcd for $\mathrm{C}_{11} \mathrm{H}_{20} \mathrm{O}_{6}$ : 248.1260. Found: 248.1263 .

exo-3,5-0-Propylidene-2,4,6-tri-0-methyl-myo-inositol (9b). In a manner similar to that described above, 8a $(0.49$ $\mathrm{g}, 2.11 \mathrm{mmol})$ in benzene $(20 \mathrm{~mL})$ was treated with $\mathrm{EtMgBr}$ (2.2 $\mathrm{mL}$ in $2.5 \mathrm{M}$ ether solution, $5.50 \mathrm{mmol}$ ) at $65^{\circ} \mathrm{C}$ for $16 \mathrm{~h}$ to afford racemic $\mathbf{9 b}$ as an oil $(0.38 \mathrm{~g}, 68 \%$ ): IR (neat) $v 3511$ $\mathrm{cm}^{-1}$; ${ }^{1} \mathrm{H}$ NMR $\left(200 \mathrm{MHz} \mathrm{CDCl}_{3}\right) \delta 0.88(\mathrm{t}, \mathrm{J}=7.5 \mathrm{~Hz}, 3 \mathrm{H})$, $1.51(\mathrm{dq}, \mathrm{J}=5.0,7.5 \mathrm{~Hz}, 2 \mathrm{H}), 3.05(\mathrm{br} \mathrm{s}, 1 \mathrm{H}), 3.34(\mathrm{~s}, 3 \mathrm{H})$, $3.71(\mathrm{dt}, \mathrm{J}=1.0,4.2 \mathrm{~Hz}, 1 \mathrm{H}), 3.82(\mathrm{dd}, \mathrm{J}=6.5,7.7 \mathrm{~Hz}, 1 \mathrm{H})$, 4.14-4.22 (m, $2 \mathrm{H}), 4.45$ (ddd, J = 1.8, 4.2, 6.5 Hz, $1 \mathrm{H}$ ), 4.97 $(\mathrm{t}, \mathrm{J}=5.0 \mathrm{~Hz}, 1 \mathrm{H}) ;{ }^{13} \mathrm{C} \mathrm{NMR}\left(50 \mathrm{MHz} \mathrm{CDCl}_{3}\right) \delta 8.0,27.9$, 56.8, 58.0, 58.5, 67.5, 68.1, 71.4, 73.9, 75.1, 84.5, 94.3; HRMS Calcd for $\mathrm{C}_{10} \mathrm{H}_{17} \mathrm{O}_{6}(\mathrm{M}-43)$ : 233.1025. Found: 233.1028 .

exo-3,5-O-Benzylidene-2,4,6-tri-0-methyl-myo-inositol (9c). In a manner similar to that described above, 8a (1.51 $\mathrm{g}, 6.51 \mathrm{mmol})$ in benzene $(30 \mathrm{~mL})$ was treated with $\mathrm{PhMgBr}$ $(4.0 \mathrm{~mL}$ in $2.1 \mathrm{M}$ ether solution, $8.4 \mathrm{mmol})$ at $65^{\circ} \mathrm{C}$ for $16 \mathrm{~h}$ to afford racemic 9c as an oil (1.32 g, 68\%): IR (neat) $v 3526$ $\mathrm{cm}^{-1}$; ${ }^{1} \mathrm{H}$ NMR $\left(400 \mathrm{MHz} \mathrm{CDCl}_{3}\right) \delta 3.25(\mathrm{~d}, \mathrm{~J}=6.4 \mathrm{~Hz}, 1 \mathrm{H})$, $3.40(\mathrm{~s}, 3 \mathrm{H}), 3.53(\mathrm{~s}, 3 \mathrm{H}), 3.69(\mathrm{~d}, \mathrm{~J}=6.4 \mathrm{~Hz}, 1 \mathrm{H}), 3.90-4.01$ $(\mathrm{m}, 2 \mathrm{H}), 4.26-4.31(\mathrm{~m}, 1 \mathrm{H}), 4.35-4.42(\mathrm{~m}, 1 \mathrm{H}), 4.62$ (ddd, J $=1.8,4.4,6.4 \mathrm{~Hz}, 1 \mathrm{H}), 6.07(\mathrm{~s}, 1 \mathrm{H}), 7.31-7.38(\mathrm{~m}, 3 \mathrm{H}), 7.43-$ $7.47(\mathrm{~m}, 2 \mathrm{H}) ;{ }^{13} \mathrm{C} \mathrm{NMR}\left(75 \mathrm{MHz}, \mathrm{CDCl}_{3}\right) \delta 57.0,58.1,58.6$, 68.1, 68.2, 71.9, 73.9, 75.0, 84.4, 93.3, 126.2, 128.3, 129.0, 138.1; HRMS Calcd for $\mathrm{C}_{16} \mathrm{H}_{22} \mathrm{O}_{6}$ : 310.1416. Found: 310.1421 .

exo-1'-Phenyl-3,5-0-ethylidene-2,4,6-tri-0-methyl-myoinositol (9d). In a manner similar to that described above, $\mathbf{8 b}(0.47 \mathrm{~g}, 1.52 \mathrm{mmol})$ in benzene $(15 \mathrm{~mL})$ was treated with MeMgl $(8.8 \mathrm{~mL}$ in $2 \mathrm{M}$ ether solution, $9.6 \mathrm{mmol})$ at $15^{\circ} \mathrm{C}$ for $16 \mathrm{~h}$ to afford racemic $9 \mathrm{~d}(0.28 \mathrm{~g}, 57 \%): \mathrm{mp} 102-104{ }^{\circ} \mathrm{C}$; I R (neat) $v 3561 \mathrm{~cm}^{-1}$; ${ }^{1 \mathrm{H}} \mathrm{NMR}\left(300 \mathrm{MHz} \mathrm{CDCl}_{3}\right) \delta 1.73(\mathrm{~s}, 3 \mathrm{H})$, $2.84(\mathrm{~d}, \mathrm{~J}=9.0 \mathrm{~Hz}, 1 \mathrm{H}), 3.43(\mathrm{~s}, 3 \mathrm{H}), 3.45(\mathrm{~s}, 6 \mathrm{H}), 3.66-3.71$ $(\mathrm{m}, 1 \mathrm{H}), 3.81(\mathrm{dd}, \mathrm{J}=1.5,5.9 \mathrm{~Hz}, 1 \mathrm{H}), 4.07$ (ddd, $\mathrm{J}=2.7$, $5.9,9.0 \mathrm{~Hz}, 1 \mathrm{H}), 4.45-4.52(\mathrm{~m}, 1 \mathrm{H}), 4.56-4.65(\mathrm{~m}, 1 \mathrm{H}), 4.73$ (br d, J $=5.6 \mathrm{~Hz}, 1 \mathrm{H}), 7.19-7.32(\mathrm{~m}, 3 \mathrm{H}), 7.47-7.51(\mathrm{~m}, 2$ $\mathrm{H}) ;{ }^{13} \mathrm{C} \mathrm{NMR}\left(75 \mathrm{MHz} \mathrm{CDCl}_{3}\right) \delta 33.6,56.8,57.6,58.7,68.1$, 68.9, 70.7, 72.6, 74.9, 86.0, 98.9, 124.2, 127.7, 128.1, 146.0; Anal. Calcd C, 62.95\%; H, 7.46\%. Found: C, 62.59\%; $\mathrm{H}$, $7.18 \%$.

endo-4,6-0-Benzylidene-exo-3,5-ethylidene-2-0-methyl-myo-inositol (11a). In a manner similar to that described above, $10(0.40 \mathrm{~g}, 1.37 \mathrm{mmol})$ in benzene $(23 \mathrm{~mL})$ was treated with $\mathrm{MeMgl}$ ( $2.8 \mathrm{~mL}$ in $2 \mathrm{M}$ ether solution, $5.6 \mathrm{mmol}$ ) at 55 ${ }^{\circ} \mathrm{C}$ for $16 \mathrm{~h}$ to afford racemic $11 \mathrm{a}(0.20 \mathrm{~g}, 47 \%)$ : IR (neat) $v$ $3457 \mathrm{~cm}^{-1}$; ${ }^{1} \mathrm{H}$ NMR $\left(200 \mathrm{MHz} \mathrm{CDCl}_{3}\right) \delta 1.26(\mathrm{~d}, \mathrm{~J}=4.7 \mathrm{~Hz}$, $3 \mathrm{H}), 2.99(\mathrm{br}, 1 \mathrm{H}), 3.54(\mathrm{~s}, 3 \mathrm{H}), 4.16(\mathrm{dd}, \mathrm{J}=4.9,7.4 \mathrm{~Hz}, 1$ $\mathrm{H}), 4.44(\mathrm{~d}, \mathrm{~J}=7.4 \mathrm{~Hz}, 1 \mathrm{H}), 4.52-4.59(\mathrm{~m}, 2 \mathrm{H}), 4.63(\mathrm{dt}, \mathrm{J}$ $=1.7,5.2 \mathrm{~Hz}, 1 \mathrm{H}), 4.81-4.90(\mathrm{~m}, 1 \mathrm{H}), 5.69(\mathrm{q}, \mathrm{J}=4.7 \mathrm{~Hz}, 1$ $\mathrm{H}), 6.04(\mathrm{~s}, 1 \mathrm{H}), 7.34-7.41(\mathrm{~m}, 5 \mathrm{H}) ;{ }^{13} \mathrm{C} \mathrm{NMR}\left(50 \mathrm{MHz}^{\mathrm{C}} \mathrm{CDCl}_{3}\right)$ $\delta 21.4,57.4,64.2,67.2,67.7,68.8,72.9,74.2,90.9,92.8,126.2$, $128.5,129.4,137.4$.

endo-4,6-0-Benzylidene-exo-3,5-ethylidene-1,2-di-0methyl-myo-inositol (11b). To a mixture of 11a $(0.19 \mathrm{~g}, 0.62$ $\mathrm{mmol})$ and solid $\mathrm{KOH}(0.14 \mathrm{~g}, 2.50 \mathrm{mmol})$ in DMSO $(5 \mathrm{~mL})$ was added Mel $(0.08 \mathrm{~mL}, 1.3 \mathrm{mmol})$ dropwise and the mixture was stirred at rt overnight, poured into water, and extracted with $\mathrm{CH}_{2} \mathrm{Cl}_{2}$. The organic layer was washed with water and brine and dried $\left(\mathrm{MgSO}_{4}\right)$. The solvent was removed in vacuo to give the residue which was chromatographed (silica gel, 2030\% EtOAc/hexane) to give racemic $\mathbf{1 1 b}(0.19 \mathrm{~g}, 94 \%): \mathrm{mp}$ 118-119 ${ }^{\circ} \mathrm{C}$; ${ }^{1} \mathrm{H}$ NMR (300 MHz, $\left.\mathrm{CDCl}_{3}\right) \delta 1.25(\mathrm{~d}, \mathrm{~J}=4.9$ $\mathrm{Hz}, 3 \mathrm{H}), 3.51(\mathrm{~s}, 3 \mathrm{H}), 3.58(\mathrm{~s}, 3 \mathrm{H}), 3.91(\mathrm{~d}, \mathrm{~J}=7.5 \mathrm{~Hz}, 1 \mathrm{H})$, $4.25(\mathrm{dd}, \mathrm{J}=4.7,7.4 \mathrm{~Hz}, 1 \mathrm{H}), 4.49(\mathrm{t}, \mathrm{J}=4.7 \mathrm{~Hz}, 1 \mathrm{H}), 4.52-$ $4.59(\mathrm{~m}, 1 \mathrm{H}), 4.61(\mathrm{td}, \mathrm{J}=2.0,5.0 \mathrm{~Hz}, 1 \mathrm{H}), 4.90(\mathrm{br} \mathrm{t}, \mathrm{J}=5.0$ $\mathrm{Hz}, 1 \mathrm{H}), 5.85(\mathrm{q}, \mathrm{J}=4.9 \mathrm{~Hz}, 1 \mathrm{H}), 6.08(\mathrm{~s}, 1 \mathrm{H}), 7.34-7.39$ $(\mathrm{m}, 5 \mathrm{H}) ;{ }^{13} \mathrm{C}$ NMR $\left(75 \mathrm{MHz} \mathrm{CDCl}_{3}\right) \delta 21.6,57.5,59.4,63.9$, 67.3, 69.4, 72.1, 73.0, 78.6, 90.6, 92.5, 126.1, 128.5, 129.5, 137.4; HRMS Calcd for $\mathrm{C}_{17} \mathrm{H}_{22} \mathrm{O}_{6}$ : 322.1416. Found: 322.1414. Anal. Calcd C, 63.33\%; H, 6.88\%. Found: C, 63.22\%; H, 6.85\%.

3-0-Methyl-5-0-(1'-phenylethyl)-2,4,6-0-methylidynescyllo-inositol (14). In a manner similar to that described in $9 \mathrm{a}, 13(51.7 \mathrm{mg}, 0.18 \mathrm{mmol})$ in benzene $(2 \mathrm{~mL})$ was treated with $\mathrm{MeMgl}(0.5 \mathrm{~mL}$ in $2 \mathrm{M}$ ether solution, $1.0 \mathrm{mmol})$ at $\mathrm{rt}$ for $16 \mathrm{~h}$ to afford a mixture of inseparable diasteromers 14 (34.0 $\mathrm{mg}, 56 \%, \mathrm{dr}=2: 1)$. The major isomer exhibited ${ }^{1} \mathrm{H}$ NMR (300 $\left.\mathrm{MHz} \mathrm{CDCl}_{3}\right)$ at $\delta 1.42(\mathrm{~d}, \mathrm{~J}=6.4 \mathrm{~Hz}, 3 \mathrm{H}), 3.52(\mathrm{~s}, 3 \mathrm{H}), 4.07-$ $4.57(\mathrm{~m}, 6 \mathrm{H}), 4.67(\mathrm{q}, \mathrm{J}=6.4 \mathrm{~Hz}, 1 \mathrm{H}), 5.40(\mathrm{~s}, 1 \mathrm{H}), 7.25-$ $7.38(\mathrm{~m}, 5 \mathrm{H})$. The minor isomer showed characteristic ${ }^{1} \mathrm{H}$ NMR absorptions at $\delta 1.43(\mathrm{~d}, \mathrm{~J}=6.4 \mathrm{~Hz}, 3 \mathrm{H})$ and $3.47(\mathrm{~s}, 3$ H).

5-O-Isopropyl-2,4,6-tri-O-benzyl-myo-inositol (15a). In a manner similar to that described in $9 a, 6(0.14 \mathrm{~g}, 0.30 \mathrm{mmol})$ in benzene $(3 \mathrm{~mL})$ was treated with $\mathrm{MeMgl}(0.45 \mathrm{~mL}$ in $2 \mathrm{M}$ ether solution, $0.90 \mathrm{mmol}$ ) under reflux for $16 \mathrm{~h}$ to afford $\mathbf{1 5 a}$ (0.11 g, 69\%): IR (neat) $v 3455 \mathrm{~cm}^{-1} ;{ }^{1} \mathrm{H}$ NMR $(400 \mathrm{MHz}$, $\left.\mathrm{CDCl}_{3}\right) \delta 1.23(\mathrm{~d}, \mathrm{~J}=6.0 \mathrm{~Hz}, 6 \mathrm{H}), 2.25(\mathrm{~d}, \mathrm{~J}=5.4 \mathrm{~Hz}, 2 \mathrm{H})$, $3.36(\mathrm{t}, \mathrm{J}=9.6 \mathrm{~Hz}, 1 \mathrm{H}), 3.50(\mathrm{ddd}, \mathrm{J}=2.4,5.6,9.6 \mathrm{~Hz}, 2 \mathrm{H})$, $3.68(\mathrm{t}, \mathrm{J}=9.6 \mathrm{~Hz}, 2 \mathrm{H}$ ), $3.96(\mathrm{t}, \mathrm{J}=2.4 \mathrm{~Hz}, 1 \mathrm{H}$ ), 4.10 (sept, $\mathrm{J}=9.6 \mathrm{~Hz}, 1 \mathrm{H}), 4.74(\mathrm{~d}, \mathrm{~J}=11.2 \mathrm{~Hz}, 2 \mathrm{H}), 4.77(\mathrm{~s}, 2 \mathrm{H}), 4.93$ $(\mathrm{d}, \mathrm{J}=11.2 \mathrm{~Hz}, 2 \mathrm{H}), 7.28-7.40(\mathrm{~m}, 15 \mathrm{H}) ;{ }^{13} \mathrm{C} \mathrm{NMR}(75 \mathrm{MHz}$, $\left.\mathrm{CDCl}_{3}\right) \delta 22.7,72.6,73.1,75.2,75.8,78.8,79.6,82.3,127.7$, $127.8,128.0,128.4,128.5,138.5$, 138.6; HRMS Calcd for $\mathrm{C}_{30} \mathrm{H}_{36} \mathrm{O}_{6}: 492.2512$. Found: 492.2510 .

5-O-Benzhydryl-2,4,6-tri-O-benzyl-myo-inositol (15b). In a manner similar to that described in $9 \mathbf{a}, \mathbf{6}(0.32 \mathrm{~g}, 0.70$ $\mathrm{mmol})$ in benzene $(7 \mathrm{~mL})$ was treated with $\mathrm{PhMgBr}(1.7 \mathrm{~mL}$ in $2 \mathrm{M}$ ether solution, $3.40 \mathrm{mmol}$ ) and was refluxed for $16 \mathrm{~h}$ to yield $15 \mathbf{b}(0.32 \mathrm{~g}, 72 \%): \mathrm{mp} 115-117^{\circ} \mathrm{C}$; IR (neat) $v 3558 \mathrm{~cm}^{-1}$; ${ }^{1} \mathrm{H}$ NMR $\left(300 \mathrm{MHz}, \mathrm{CDCl}_{3}\right) \delta 2.24(\mathrm{~d}, \mathrm{~J}=5.3 \mathrm{~Hz}, 2 \mathrm{H}), 3.48$ $(\mathrm{ddd}, \mathrm{J}=2.8,5.3,9.2 \mathrm{~Hz}, 2 \mathrm{H}), 3.58(\mathrm{t}, \mathrm{J}=9.2 \mathrm{~Hz}, 1 \mathrm{H}), 3.83$ $(\mathrm{t}, \mathrm{J}=9.2 \mathrm{~Hz}, 2 \mathrm{H}), 3.94(\mathrm{t}, \mathrm{J}=2.8 \mathrm{~Hz}, 1 \mathrm{H}), 4.73(\mathrm{~s}, 4 \mathrm{H})$, $4.76(\mathrm{~s}, 2 \mathrm{H}), 6.13(\mathrm{~s}, 1 \mathrm{H}), 7.18-7.39(\mathrm{~m}, 25 \mathrm{H}) ;{ }^{13} \mathrm{C}$ NMR $(75$ $\left.\mathrm{MHz}_{2} \mathrm{CDCl}_{3}\right) \delta 72.6,75.2,75.5,78.3,78.7,82.4,83.5,127.3$, $127.4,127.8,128.0,128.1,128.2,128.5,138.4,142.6$; HRMS Calcd for $\mathrm{C}_{40} \mathrm{H}_{42} \mathrm{O}_{6}$ : 618.2981. F ound: $525.2232\left(\mathrm{M}^{+}-93\right)$. Anal. Calcd C, 77.63\%; H, 6.85\%. Found: C, 77.54\%, H, $6.51 \%$.

5-0-Benzhydryl-2,4,6-tri-O-methyl-myo-inositol (15c). In a manner similar to that described in 9a, 8a $(0.36 \mathrm{~g}, 1.53$ $\mathrm{mmol})$ in benzene $(15 \mathrm{~mL})$ was treated with $\mathrm{PhMgBr}(3.1 \mathrm{~mL}$ in $2.0 \mathrm{M}$ ether solution, $6.2 \mathrm{mmol}$ ) under reflux for $16 \mathrm{~h}$ to give $15 \mathrm{c}(0.24 \mathrm{~g}, 60 \%)$ : $\mathrm{mp} 89-90^{\circ} \mathrm{C}$; IR (neat) $v 3420 \mathrm{~cm}^{-1}$; ${ }^{1} \mathrm{H} \mathrm{NMR}\left(300 \mathrm{MHz} \mathrm{CDCl}_{3}\right) \delta 2.39(\mathrm{~d}, \mathrm{~J}=5.1 \mathrm{~Hz}, 2 \mathrm{H}), 3.29$ $2.52(\mathrm{~m}, 5 \mathrm{H}), 3.48(\mathrm{~s}, 6 \mathrm{H}), 3.63(\mathrm{~s}, 3 \mathrm{H}), 3.67(\mathrm{t}, \mathrm{J}=2.5 \mathrm{~Hz}$, $1 \mathrm{H}), 5.95(\mathrm{~s}, 1 \mathrm{H}), 7.18-7.39(\mathrm{~m}, 10 \mathrm{H}) ;{ }^{13} \mathrm{C} N M R(75 \mathrm{MHz}$, $\left.\mathrm{CDCl}_{3}\right) \delta 61.3,61.7,72.6,78.4,80.4,83.8,84.0,127.3,127.4$, 128.1, 142.7; HRMS Calcd for $\mathrm{C}_{22} \mathrm{H}_{28} \mathrm{O}_{6}$ : 388.1886. Found: 388.1885. Anal. Calcd C, 68.01\%; H, 7.27\%. Found: C, $67.74 \% ; \mathrm{H}, 7.15 \%$.

1,3-O-Diacetyl-5-0-benzhydryl-2,4,6-tri-0-methyl-myoinositol (16). A benzene solution $(10 \mathrm{~mL})$ of $15 \mathrm{c}(0.97 \mathrm{~g}, 2.49$ $\mathrm{mmol}), \mathrm{Ac}_{2} \mathrm{O}(0.58 \mathrm{~mL})$, and pyridine $(0.5 \mathrm{~mL})$ was refluxed for $5 \mathrm{~h}$. The mixture was cooled, diluted with ether, and washed with $10 \% \mathrm{HCl}$ and saturated $\mathrm{NaCl}$. The organic solution was dried $\left(\mathrm{MgSO}_{4}\right)$, filtered, and concentrated. The residue was chromatographed (silica gel, 30\% EtOAc/hexane) to afford $16(0.84 \mathrm{~g}, 71 \%)$ : $\mathrm{mp} 135-137{ }^{\circ} \mathrm{C}$; IR (neat) $v 1751$ $\mathrm{cm}^{-1}$; ${ }^{1} \mathrm{H}$ NMR $\left(300 \mathrm{MHz}, \mathrm{CDCl}_{3}\right) \delta 2.08(\mathrm{~s}, 6 \mathrm{H}), 3.35(\mathrm{~s}, 6 \mathrm{H})$, $3.42(\mathrm{t}, \mathrm{J}=9.0 \mathrm{~Hz}, 1 \mathrm{H}), 3.47(\mathrm{~s}, 3 \mathrm{H}), 3.64(\mathrm{dd}, \mathrm{J}=9.1,10.1$ $\mathrm{Hz}, 2 \mathrm{H}), 3.71(\mathrm{t}, \mathrm{J}=2.5 \mathrm{~Hz}, 1 \mathrm{H}), 4.67(\mathrm{dd}, \mathrm{J}=2.5,10.1 \mathrm{~Hz}$, $2 \mathrm{H}), 5.94(\mathrm{~s}, 1 \mathrm{H}), 7.17-7.39(\mathrm{~m}, 10 \mathrm{H}) ;{ }^{13} \mathrm{C} N M R(75 \mathrm{MHz}$, $\left.\mathrm{CDCl}_{3}\right) \delta 20.9,60.9,61.3,73.5,78.0,78.8,81.1,84.4,127.2$, $127.3,128.0,142.7,169.9$; HRMS Calcd for $\mathrm{C}_{26} \mathrm{H}_{32} \mathrm{O}_{8}: 472.2097$. Found: 472.2109. Anal. Calcd C, 66.09\%; H, 6.83\%. Found: C, $66.06 \% ; \mathrm{H}, 6.83 \%$.

1,3-O-Diacetyl-2,4,6-tri-O-methyl-myo-inositol (17). A methanolic solution $(100 \mathrm{~mL})$ of $\mathbf{1 6}(0.42 \mathrm{~g}, 0.89 \mathrm{mmol})$ in the presence of $\mathrm{Pd} / \mathrm{C}(5 \%, 83 \mathrm{mg})$ was treated with $\mathrm{H}_{2}$ at $1 \mathrm{~atm}$ for $4 \mathrm{~h}$. The mixture was filtered on Celite, and the filtrate was evaporated in vacuo to give the residue which was chromatographed (silica gel, 50\% EtOAc/hexane) to afford $\mathbf{1 7}$ 
(0.23 g, 86\%): $\mathrm{mp} 113-114{ }^{\circ} \mathrm{C}$; IR (neat) $v 3561,1746 \mathrm{~cm}^{-1}$; ${ }^{1} \mathrm{H}$ NMR $\left(300 \mathrm{MHz} \mathrm{CDCl}_{3}\right) \delta 2.14(\mathrm{~s}, 6 \mathrm{H}), 2.65(\mathrm{~d}, \mathrm{~J}=2.4$ $\mathrm{Hz}, 1 \mathrm{H}), 3.40-3.56(\mathrm{~m}, 4 \mathrm{H}), 3.47(\mathrm{~s}, 3 \mathrm{H}), 3.54(\mathrm{~s}, 3 \mathrm{H}), 3.76$ $(\mathrm{t}, \mathrm{J}=2.8 \mathrm{~Hz}, 1 \mathrm{H}), 4.75(\mathrm{dd}, \mathrm{J}=2.4,9.6 \mathrm{~Hz}, 1 \mathrm{H}) ;{ }^{13} \mathrm{C} \mathrm{NMR}$ $\left(100 \mathrm{MHz} \mathrm{CDCl}_{3}\right) \delta 21.0,60.8,61.6,73.3,74.2,78.4,80.2$, 169.9; HRMS Calcd for $\mathrm{C}_{13} \mathrm{H}_{22} \mathrm{O}_{8}$ : 306.1315. Found: 306.1317 . Anal. Calcd C, 50.96\%; H, 7.24\%. Found: C, 51.15\%; H, $7.30 \%$.

2,3,4,6-Tetra-0-methyl-myo-inositol (18). To a mixture of $9 \mathrm{a}(1.09 \mathrm{~g}, 4.40 \mathrm{mmol})$ and solid $\mathrm{KOH}(1.01 \mathrm{~g}, 18.0 \mathrm{mmol})$ in DMSO $(20 \mathrm{~mL})$ was added Mel $(0.7 \mathrm{~mL}, 11.2 \mathrm{mmol})$ dropwise, and the mixture was stirred at rt overnight, poured into water, and extracted with $\mathrm{CH}_{2} \mathrm{Cl}_{2}$. The organic layer was washed with water and brine and dried $\left(\mathrm{MgSO}_{4}\right)$. The solvent was removed in vacuo to give the residue which was chromatographed (silica gel, 50\% EtOAc/hexane) to give the corresponding ether as an oil: ${ }^{1} \mathrm{H}$ NMR $\left(300 \mathrm{MHz} \mathrm{CDCl}_{3}\right) \delta$ $1.21(\mathrm{~d}, \mathrm{~J}=4.8 \mathrm{~Hz}, 3 \mathrm{H}), 3.35(\mathrm{~s}, 3 \mathrm{H}), 3.47(\mathrm{~s}, 6 \mathrm{H}), 3.49(\mathrm{~s}, 3$ $\mathrm{H}), 3.57(\mathrm{~d}, \mathrm{~J}=6.9 \mathrm{~Hz}, 1 \mathrm{H}), 3.70-3.74(\mathrm{~m}, 1 \mathrm{H}), 3.82(\mathrm{t}, \mathrm{J}=$ $7.5 \mathrm{~Hz}, 1 \mathrm{H}), 3.93(\mathrm{dd}, \mathrm{J}=6.9,7.5 \mathrm{~Hz}, 1 \mathrm{H}), 4.16(\mathrm{dd}, \mathrm{J}=1.8$, $3.5 \mathrm{~Hz}, 1 \mathrm{H}$ ), 4.46 (ddd, J = 1.8, 4.0,6.3 Hz, $1 \mathrm{H}$ ), $5.19(\mathrm{q}, \mathrm{J}=$ $4.8 \mathrm{~Hz}, 1 \mathrm{H}) ;{ }^{13} \mathrm{C} N M R\left(75 \mathrm{MHz} \mathrm{CDCl}_{3}\right) \delta 20.7,56.9,57.8$, 58.1, 58.6, 67.8, 71.9, 72.2, 75.0, 76.6, 82.5, 90.7; HRMS Calcd for $\mathrm{C}_{12} \mathrm{H}_{22} \mathrm{O}_{6}$ : 262.1416. Found: 262.1410 .

A mixture of the ether $(0.32 \mathrm{~g}, 1.23 \mathrm{mmol})$ and $\mathrm{TsOH}(58$ $\mathrm{mg}, 0.20 \mathrm{mmol})$ in $\mathrm{MeOH}(10 \mathrm{~mL})$ was stirred at rt for $6 \mathrm{~h}$ and then treated with solid $\mathrm{K}_{2} \mathrm{CO}_{3}$. After filtration, the filtrate was concentrated, and the racemic $\mathbf{1 8}$ was crystallized from the filtrate $(0.24 \mathrm{~g}, 76 \%): \mathrm{mp} 144-146{ }^{\circ} \mathrm{C}$; IR (neat) $v 3451$ $\mathrm{cm}^{-1}$; ${ }^{1} \mathrm{H}$ NMR $\left(400 \mathrm{MHz}, \mathrm{D}_{2} \mathrm{O}+\mathrm{DMSO}^{-\mathrm{d}_{6}}\right) \delta 2.95-3.11(\mathrm{~m}, 4$ H), $3.20-3.23(\mathrm{dd}, \mathrm{J}=2.4,9.6 \mathrm{~Hz}, 1 \mathrm{H}), 3.29(\mathrm{~s}, 3 \mathrm{H}), 3.37(\mathrm{~s}$,
$3 \mathrm{H}), 3.40(\mathrm{~s}, 3 \mathrm{H}), 3.59(\mathrm{t}, \mathrm{J}=2.4 \mathrm{~Hz}, 1 \mathrm{H}) ;{ }^{13} \mathrm{C} \mathrm{NMR}(100$ MHz, DMSO-d 6 ) $\delta 57.9,59.8,59.9,70.9,73.9,79.4,81.4,82.9$, 83.5; HRMS Calcd for $\mathrm{C}_{10} \mathrm{H}_{20} \mathrm{O}_{6}$ : 236.1260. Found: 236.1264.

3-O-Benzyl-2,4,6-tri-O-methyl-myo-inositol (19). Compound $\mathbf{8 b}(1.14 \mathrm{~g}, 3.70 \mathrm{mmol})$ in $\mathrm{CH}_{2} \mathrm{Cl}_{2}(10 \mathrm{~mL})$ was treated with $\mathrm{LiAlH}_{4}(0.17 \mathrm{~g})$ in the presence of a catalytic amount of $\mathrm{AlCl}_{3}$ (ca. $\left.50 \mathrm{mg}\right)$ in $\mathrm{Et}_{2} \mathrm{O}(30 \mathrm{~mL})$. The mixture was refluxed for $16 \mathrm{~h}$, diluted with ether, washed with saturated $\mathrm{NaCl}$, dried $\left(\mathrm{MgSO}_{4}\right)$, filtered, and concentrated. The residue was chromatographed (silica gel, 30-50\% EtOAc/hexane) to afford racemic $19(0.57 \mathrm{~g}, 49 \%): \mathrm{mp} 124-125^{\circ} \mathrm{C}$; IR (neat) $v 3428$ $\mathrm{cm}^{-1}$; ${ }^{1} \mathrm{H}$ NMR $\left(300 \mathrm{MHz} \mathrm{CDCl}_{3}\right) \delta 2.46(\mathrm{~d}, \mathrm{~J}=6.0 \mathrm{~Hz}, 1 \mathrm{H})$, $2.67(\mathrm{br}, 1 \mathrm{H}), 3.26-3.39(\mathrm{~m}, 4 \mathrm{H}), 3.44-3.51(\mathrm{~m}, 1 \mathrm{H}), 3.63(\mathrm{~s}$, $3 \mathrm{H}), 3.64(\mathrm{~s}, 3 \mathrm{H}), 3.65(\mathrm{~s}, 3 \mathrm{H}), 3.73(\mathrm{t}, \mathrm{J}=2.3 \mathrm{~Hz}, 1 \mathrm{H}), 4.67$

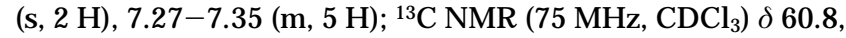
$61.4,61.5,72.1,72.6,74.6,79.2,80.7,82.6,82.8,127.6,127.8$, 128.4, 138.0; HRMS Calcd for $\mathrm{C}_{16} \mathrm{H}_{24} \mathrm{O}_{6}$ : 312.1573. Found: 312.1578. Anal. Calcd C, 61.52\%; H, 7.74\%. Found: C, $61.27 \% ; \mathrm{H}, 7.74 \%$.

Acknowledgment. This work was supported by the National Science Council of the Republic of China.

Supporting Information Available: NMR spectra for 9a-d, 11a, 11b, 15a-c, and 16-19 and ORTEP of 9d and 11b (14 pages). This material is contained in libraries on microfiche, immediately follows this article in the microfilm version of the journal, and can be ordered from the ACS; see any current masthead page for ordering information. 